\title{
The spectroscopic Hertzsprung-Russell diagram of Galactic massive stars ${ }^{\star}$
}

\author{
N. Castro ${ }^{1}$, L. Fossati ${ }^{1}$, N. Langer ${ }^{1}$, S. Simón-Díaz ${ }^{2,3}$, F. R. N. Schneider ${ }^{1}$, and R. G. Izzard ${ }^{1}$ \\ 1 Argelander-Institut für Astronomie der Universität Bonn, Auf dem Hügel 71, 53121 Bonn, Germany \\ e-mail: norberto@astro.uni-bonn.de \\ 2 Instituto de Astrofísica de Canarias, 38200 La Laguna, Tenerife, Spain \\ 3 Universidad de La Laguna, 38205 La Laguna, Tenerife, Spain \\ Received 19 September 2014 / Accepted 7 October 2014
}

ABSTRACT

\begin{abstract}
The distribution of stars in the Hertzsprung-Russell diagram narrates their evolutionary history and directly assesses their properties. Placing stars in this diagram however requires the knowledge of their distances and interstellar extinctions, which are often poorly known for Galactic stars. The spectroscopic Hertzsprung-Russell diagram (sHRD) tells similar evolutionary tales, but is independent of distance and extinction measurements. Based on spectroscopically derived effective temperatures and gravities of almost 600 stars, we derive for the first time the observational distribution of Galactic massive stars in the sHRD. While biases and statistical limitations in the data prevent detailed quantitative conclusions at this time, we see several clear qualitative trends. By comparing the observational sHRD with different state-of-the-art stellar evolutionary predictions, we conclude that convective core overshooting may be massdependent and, at high mass $\left(\gtrsim 15 M_{\odot}\right)$, stronger than previously thought. Furthermore, we find evidence for an empirical upper limit in the sHRD for stars with $T_{\text {eff }}$ between 10000 and $32000 \mathrm{~K}$ and, a strikingly large number of objects below this line. This over-density may be due to inflation expanding envelopes in massive main-sequence stars near the Eddington limit.
\end{abstract}

Key words. stars: evolution - Hertzsprung-Russell and C-M diagrams - stars: massive

\section{Introduction}

The Hertzsprung-Russell diagram (HRD, Hertzsprung 1905; Russell 1919) is probably the most important tool for analysing stellar evolution (Nielsen 1964). The main-sequence is the most prominent feature in the HRD, identifying the core hydrogen burning stars and allowing us to distinguish the later evolutionary stages (e.g. Massey 2003; Soderblom 2010). However, the stellar distance and reddening are required to derive the stellar luminosity, which may render uncertain the position of, e.g., Galactic stars in the HRD. Langer \& Kudritzki (2014) proposed an alternative tool for analysing physical properties of observed stars and testing stellar evolution models, the spectroscopic Hertzsprung-Russell diagram (sHRD). The sHRD is obtained from the HRD by replacing the luminosity $(L)$ to the quantity $\mathscr{L}:=T_{\text {eff }}^{4} / g$, which is the inverse of the flux-weighted gravity introduced by Kudritzki et al. (2003). The value of $\mathscr{L}$ can be calculated from stellar atmosphere analyses without prior knowledge of the distance or the extinction. In contrast to the classical $T_{\text {eff }}-\log g$ diagram (Kiel diagram), the sHRD sorts stars according to their proximity to the Eddington limit, because $\mathscr{L}$ is proportional to the Eddington factor $\Gamma_{\mathrm{e}}=L / L_{\mathrm{Edd}}$,

$\mathscr{L}=\frac{1}{4 \pi \sigma G} \frac{L}{M}=\frac{c}{\sigma \kappa_{\mathrm{e}}} \Gamma_{\mathrm{e}}$,

where $\sigma$ is the Stefan-Boltzmann constant, $\kappa_{\mathrm{e}}$ is the electron scattering opacity in the stellar envelope, and the other symbols have their usual meanings.

Except for their final stages, the evolution of low and intermediate mass stars, including our Sun, is well understood

\footnotetext{
* Appendix $\mathrm{A}$ is available in electronic form at http://www . aanda.org
}

(e.g. Vandenberg 1985; Girardi et al. 2000), as their initial mass largely settles their evolution and fate (Iben 1967). In massive $\left(\gtrsim 8 M_{\odot}\right)$ stars, however, even the core hydrogen burning phase is not yet well understood and the knowledge about core helium burning evolution stages is even more uncertain (Langer 2012). The higher the considered masses the less we know about how the stars evolve. The main reason for this is that, besides their initial mass, the evolution of massive stars depends on further initial parameters: spin (Meynet \& Maeder 2000; Heger et al. 2000) and duplicity (Sana et al. 2012). Furthermore, uncertain mass loss rates (Smith 2014) and internal mixing processes (Bressan et al. 1981; Langer 1991; Schaller et al. 1992; Yoon et al. 2006) are more important the more massive the stars. For these reasons, our knowledge of the evolution of massive stars is rudimentary and observational constraints are urgently needed.

To exploit the capacities of the sHRD for massive stars, we have mined the available quantitative spectroscopic studies in the literature, and augmented this with our own analyses of luminous $\mathrm{OB}$ stars, to collect accurate effective temperatures and gravities of a large number of Galactic massive stars. This allows us to establish for the first time the distribution of these objects in the sHRD. After discussing our stellar sample and its biases in Sect. 2, we show an empirical sHRD in Sect. 3, where we also compare it with predictions from recent stellar evolution models. In Sect. 4, we discuss our results and present conclusions.

\section{Stellar sample}

Our sample comprises 575 stars, 439 of which have $T_{\text {eff }} \geq$ $10000 \mathrm{~K}$ and $\log \mathscr{L} / \mathscr{L}_{\odot}>2.75$ (corresponding to masses above $\sim 9 M_{\odot}$ ). The analyses of 255 stars belong to the IACOB spectroscopic survey of Northern Galactic OB-stars project 


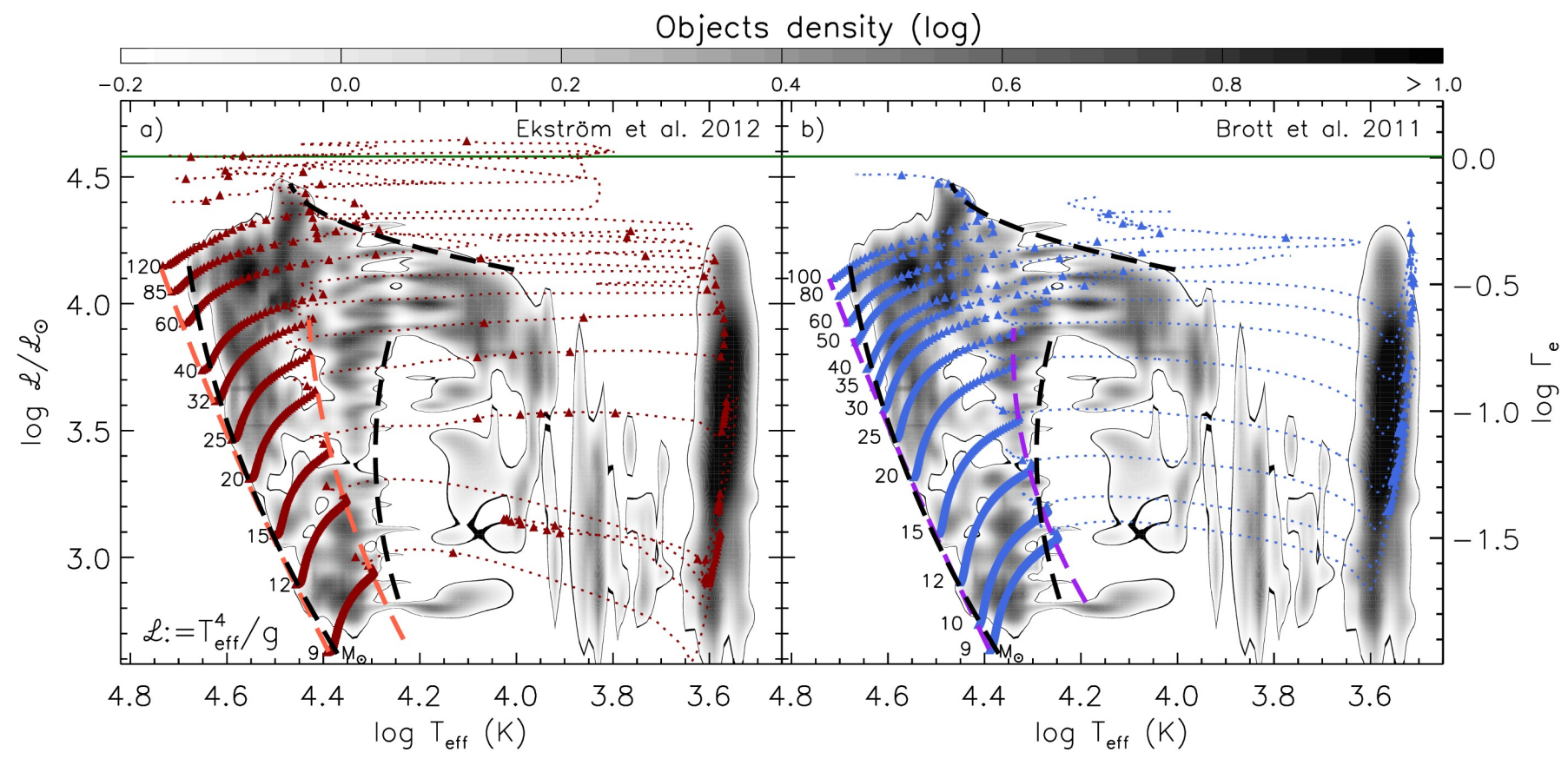

Fig. 1. Grey scale representation of the probability density distribution of the location of 575 Galactic stars in the sHRD. Three empirical borderlines between densely populated regions and empty regions are drawn as black dashed lines (cf. Table 1). The electron scattering Eddington factor $\left(\Gamma_{\mathrm{e}}\right)$ is given on the right ordinate axis. The $\Gamma_{\mathrm{e}}$ limit for hydrogen-rich composition at $\log \mathscr{L} / \mathscr{L}_{\odot}=4.6$ is represented by a green horizontal line. Overlayed are stellar evolution tracks for non-rotating stars with solar composition: a) Ekström et al. (2012) and, b) Brott et al. (2011). The ZAMS and TAMS positions of the models are connected through orange and purple dashed lines. Red and blue triangles are placed on the tracks separated by $0.1 \mathrm{Myr}$.

(Simón-Díaz et al. 2011a; Simón-Díaz 2014), using the techniques described in Simón-Díaz et al. (2011b) and Castro et al. (2012). Parameters for another 250 stars were extracted from the literature (McErlean et al. 1999; Przybilla et al. 2001; Markova et al. 2004, 2014; Repolust et al. 2004; Levesque et al. 2005; Martins et al. 2005, 2012; Mokiem et al. 2005; Crowther et al. 2006; Briquet \& Morel 2007; Lefever et al. 2007, 2010; Searle et al. 2008; Hunter et al. 2009; Simón-Díaz 2010; Firnstein \& Przybilla 2012; Nieva 2013; Aerts et al. 2014). We complemented the sample with objects from the catalogue gathered by Soubiran et al. (2010) with known metallicity above $[\mathrm{Fe} / \mathrm{H}]=-0.5$.

\subsection{Completeness and biases}

The collated data comprise a heterogeneous sample in terms of instruments used for the observations, tools, and methodologies adopted for the spectral analysis. The most prominent characteristic of our sample is that it is dominated by bright stars. For instance, Soubiran et al. (2010) pointed out that $90 \%$ of the stars listed in their catalogue are brighter than $V=9.75 \mathrm{mag}$. The analysis of OB stars is also dominated by bright stars in the solar neighbourhood.

One important bias that affects the sample is the authors' interest: different authors focus on a particular kind of star to tackle a particular astrophysical topic. This leads to a spurious overpopulation of stars in certain regions (e.g. pulsational instability domains, abundances in early B-type stars or the interest in the O-type and early B-supergiant regimes) compared to others.

This melting pot approach introduces biases that cannot be quantified. Nevertheless, the sample has its strength in the large number of stars that it contains. Although it is far from giving a statistical view of the Milky Way, it is large enough to minimise some of the biases and to enable the determination of well populated regions and its borders across the upper SHRD, with the aim of constraining stellar evolution models.

\section{The sHRD of massive stars}

We determine the position of each star in the sHRD on the basis of its atmospheric parameters. We derive the probability density function by adding the Gaussian probability distributions of the stellar parameters, which are derived from their reported uncertainties. In cases where the uncertainties are not provided, we adopted typical error bars based on the studies compiled in this work. For each star, the probability distribution was calculated adopting a Gaussian distribution in the $\log \mathscr{L} / \mathscr{L}_{\odot}-\log T_{\text {eff }}$ parameter space. All distributions are summed to give the density map shown in Fig. 1. In addition, Fig. 1 shows stellar evolution tracks for non-rotating, solar-metallicity single stars published by Brott et al. (2011) and Ekström et al. (2012), and polynomial fits to the theoretical zero age main-sequence (ZAMS) and the terminal age main-sequence (TAMS) lines.

While the probability density map is somewhat patchy, it allows us to identify several borderlines that separate regions of high density from those where stars are almost absent. Three such lines are drawn in Fig. 1. One nearly vertical borderline identifies the location to the left of which no stars are found (note that Wolf-Rayet stars were not included in our sample), which could therefore relate to the ZAMS. A second nearly vertical borderline, located at $\log T_{\text {eff }}(\mathrm{K}) \simeq 4.3$, could be the TAMS. Finally, there is a close to horizontal borderline near $\log \mathscr{L} / \mathscr{L}_{\odot} \simeq 4.3$, which corresponds to an electron scattering Eddington factor of $\Gamma_{\mathrm{e}} \simeq 0.5$. These shown borderlines are quadratic fits (Table 1). Figure 1 shows an underdensity of stars across the main-sequence in the $15-25 M_{\odot}$ range. We ascribe 
Table 1. Polynomial coefficients of the observed and theoretical boundaries marked in Fig. 1.

\begin{tabular}{lrrrrr}
\hline \hline Empirical & $Y_{\min }$ & $Y_{\max }$ & $a$ & $b$ & $c$ \\
\hline ZAMS & 2.62 & 4.19 & 2.973 & 0.743 & -0.080 \\
TAMS & 2.84 & 3.88 & 2.618 & 0.982 & -0.144 \\
UPPER & 4.13 & 4.48 & -67.323 & 31.938 & -3.552 \\
\hline \multicolumn{7}{l}{ Ekström et al. (2012) (non-rotating) } \\
\hline ZAMS & 2.63 & 4.14 & 3.513 & 0.399 & -0.025 \\
TAMS & 2.68 & 3.94 & 2.816 & 0.784 & -0.095 \\
\hline \multicolumn{7}{l}{ Brott et al. (2011) (non-rotating) } \\
ZAMS & 2.64 & 4.10 & 3.586 & 0.355 & -0.019 \\
TAMS & 2.82 & 3.91 & 2.103 & 1.174 & -0.154 \\
\hline
\end{tabular}

Notes. $\log T_{\text {eff }}(K)=c Y^{2}+b Y+a$, where $Y=\log \mathscr{L} / \mathscr{L}_{\odot} . Y_{\min }$ and $Y_{\max }$ set the boundaries of quadratic fits.

this anomaly to an observational bias, but additional observations in this region are necessary.

\subsection{The mass range $8-30 M_{\odot}$}

The 8-30 $M_{\odot}$ range covers stars with spectral types in the mainsequence between B2 and O7. Figure 1 shows a good match between the observed ZAMS and both sets of evolutionary tracks. Note that, although the initial solar composition adopted by Brott et al. (2011) and Ekström et al. (2012) is slightly different, it is the iron abundance with which the opacities are interpolated in the Brott et al. (2011) models. Since the adopted iron abundances are the same, the positions of the two ZAMS lines are nearly identical.

The stars are mainly clustered on the main-sequence, where they are expected to remain for most of their lives. Our density map shows a rather tight boundary on the cool side of the mainsequence, which could be interpreted as the empirical position of the TAMS. In the following, we will use this interpretation as our working hypothesis.

The width of the main-sequence band predicted by Ekström et al. (2012) fits the observations well around $8 M_{\odot}$, but the observed main-sequence becomes wider with increasing mass up to $30 M_{\odot}$. This is not predicted by Ekström et al. (2012). On the other hand, Brott et al. (2011) predict a main-sequence that is too wide around $8 M_{\odot}$, fits well around $\sim 15 M_{\odot}$ and then worse at higher mass.

The difference between the evolutionary models might be caused by the different calibration of the convective core overshooting parameter $d_{\text {over }}$. The value of $d_{\text {over }} / H_{\mathrm{P}}=0.10$ adopted by Ekström et al. (2012) was calibrated using tracks for rotating stars in an intermediate mass range from 1.35 to $8 M_{\odot}$. On the other hand, $d_{\text {over }} / H_{\mathrm{P}}=0.335$ was used by Brott et al. (2011), as derived by comparing stars around $16 M_{\odot}$. Figure 1 shows that even more overshooting may be necessary at higher mass. In fact, Fig. 1 argues for a mass-dependent overshooting.

Figure 1 shows the tracks of non-rotating models, but using tracks for rotating stars does not solve the discrepancy (Sect. 3.3). It is possible that the mass-dependence of the discrepancy between the observed and theoretical TAMS lines is due to yet unconsidered physics, possibly connected to rotation, which is not included in either set of tracks, rather than massdependent overshooting.

On the red side of the TAMS, Fig. 1 shows a gap between the TAMS and blue supergiants of $\log T_{\text {eff }}(\mathrm{K}) \lesssim 4.15$. This post main-sequence gap, known as Hertzsprung gap, is in agreement with stellar tracks, which predict that stars rush through this gap in a fast evolution. The stars found at $\log T_{\text {eff }}(\mathrm{K}) \lesssim 4.15$ can be interpreted as core helium burning objects as predicted by models of Ekström et al. (2012). Note that the models of Brott et al. (2011) were stopped before the tracks reached this evolutionary phase. The number of objects detected in this region of our empirical SHRD could be affected by interest bias because of the presence of pulsations (e.g. Briquet \& Morel 2007).

The region covered by the red supergiant stars is reproduced by both sets of models. While the empirical temperature scale of the red supergiants may be hotter than previously thought, it is still uncertain (Levesque et al. 2005; Davies et al. 2013). In the stellar models, it is mainly the mixing length parameter that controls the temperature of the Hayashi line (Kippenhahn \& Weigert 1990).

\subsection{Stars above $30 M_{\odot}$}

The high mass part of both theoretical ZAMS lines has a clear offset from the empirical ZAMS, which increases with mass. While we cannot exclude bias effects, as the number of observed very massive stars near the ZAMS is small, the complete absence of stars near the upper end of the theoretical ZAMS lines might also be related to the fact that such young massive stars are likely still embedded in their birth clouds (Yorke 1986).

In the $\log T_{\text {eff }}(\mathrm{K}) \simeq 4.0-4.5$ range, the observations set an upper boundary in the sHRD (Table 1), reminiscent of the Humphreys-Davidson limit in the HRD (Humphreys \& Davidson 1979). We recall here that we did not include luminous blue variable stars or stars with emission line dominated spectra in our sample. Figure 1 shows that the tracks of Brott et al. (2011) fit the upper boundary of the main-sequence massive stars $\left(\log T_{\text {eff }}(\mathrm{K}) \simeq 4.3-4.5\right)$, which was interpreted as an effect of the true Eddington limit by Langer (2012; rather than the electron scattering Eddington limit).

The green line in Fig. 1 corresponds to the electron scattering Eddington limit for solar hydrogen abundance. Stars with a helium-rich surface may be located above the line, because the Eddington limit for pure helium composition lies 0.23 dex above the green line. The Ekström et al. (2012) models extend to this region because they include the Wolf-Rayet phases.

Figure 1 shows that the red supergiant branch stretches up to larger Eddington factors than the blue supergiant region. This may occur because in red supergiants a significant fraction of stellar luminosity is transported by convection rather than by radiation even out to the photospheric layers. They can thus bare larger Eddington factors even if the opacities are larger than in blue supergiant envelopes.

Below the line related to the Eddington limit, stars are distributed continuously from the main-sequence to surface temperatures just below $10000 \mathrm{~K}$. In the mass range considered here, a TAMS line cannot be identified from the data. This could imply that the main-sequence band above $30 M_{\odot}$ extends to temperatures below $10000 \mathrm{~K}$. This is predicted by the tracks of Brott et al. (2011), even though at slightly larger mass-to-luminosity ratios, and is found to be a consequence of the so called envelope inflation that occurs as a result of the proximity to the Eddington limit (Köhler et al. 2014). Alternatively, this population of blue super- and hypergiants might consist of core helium burning stars (see Langer \& Maeder 1995). Whereas their large number may be in conflict with this interpretation, we cannot rule out that some interest bias affects the observed distribution, 
because of the work done to model A- and B-supergiants (e.g. Firnstein \& Przybilla 2012).

\subsection{Rotation}

Even during the main-sequence phase, the evolution of massive stars may be significantly affected by rotation (Meynet \& Maeder 2000; Heger et al. 2000). As our sample is composed of stars with a large variety of rotational velocities, it could be important to consider stellar tracks for rotating stars also. We therefore show in Fig. A.1 a comparison of tracks for non-rotating and rapidly rotating tracks from the same model grids in Fig. 1. We do not show models with even faster rotation, because these stars are rare (Conti \& Ebbets 1977; Simón-Díaz \& Herrero 2014).

While the effects of rotation on the position of the ZAMS are negligible within the scope of our paper, the TAMS positions of the rapidly rotating models are indeed somewhat shifted with respect to the non-rotating models. However, when compared to the observational data, the conclusions drawn in the previous subsection remain unaltered. In the Ekström et al. (2012) models, the TAMS line for stars below $30 M_{\odot}$ changes very little, and for more massive stars the main-sequence band becomes even narrower with rotation. In the Brott et al. (2011) models, the main-sequence band widens for stars below $15 M_{\odot}$, which increases the discrepancy with observations. Overall, considering rapid rotation appears not to bring the models into any better agreement with the observations.

\section{Conclusions}

We present the first observational spectroscopic HertzsprungRussell diagram (sHRD) for Galactic massive stars $\left(\gtrsim 8 M_{\odot}\right)$, based on the spectroscopic analysis and atmospheric modelling of a sample of 575 stars. We produce a probability density map of the positions of stars in the sHRD. This map shows several clear borderlines that provide stringent constraints on massive star evolution models. Most notably, these lines may correspond to the ZAMS and TAMS borders of the main-sequence band. Additionally we find a sharp upper limit of observed stars in the sHRD near but somewhat below the electron scattering Eddington limit.

Except for the most massive stars, for which early hydrogen burning evolution may be hidden by their birth cocoons, the models represent the ZAMS position in the SHRD well. However, neither the models of Brott et al. (2011) nor those by Ekström et al. (2012) can reproduce what we tentatively identify as the TAMS in the sHRD. One possible interpretation is that the convective core overshooting parameter in massive stars increases with mass.

A further striking feature in our probability map is a well populated area just below the sharp upper mass-to-luminosity ratio limit in the sHRD that extends from the main-sequence to beneath $10000 \mathrm{~K}$. While none of the studied stellar evolution models can fully reproduce these stars, they may be interpreted as stars with inflated envelopes because of their proximity to the Eddington limit in the frame of the Brott et al. (2011) models.

We consider this work to be a first step towards providing essential constraints for the evolution of massive and very massive stars. Indeed, we are far from a complete view of the Milky Way stellar content, and our sample contains voids that require additional data. Several on-going surveys of OB-type stars will increase the number of objects in the SHRD in the next few years and allow us a more detailed and robust comparison with stellar evolution models.

Acknowledgements. The authors thank the referee for useful comments and helpful suggestions that improved this manuscript. L.F. and R.G.I. acknowledge financial support from the Alexander von Humboldt Foundation. S.S-.D. thanks funding from the Spanish Government Ministerio de Economia y Competitividad (MINECO) through grants AYA2010-21697-C05-04, AYA201239364-C02-01 and Severo Ochoa SEV-2011-0187, and the Canary Islands Government under grant PID2010119. F.R.N.S. acknowledges the fellowship awarded by the Bonn-Cologne Graduate School of Physics and Astronomy.

\section{References}

Aerts, C., Molenberghs, G., Kenward, M. G., \& Neiner, C. 2014, ApJ, 781, 88 Bressan, A. G., Chiosi, C., \& Bertelli, G. 1981, A\&A, 102, 25

Briquet, M., \& Morel, T. 2007, Commun. Asteroseismol., 150, 183

Brott, I., de Mink, S. E., Cantiello, M., et al. 2011, A\&A, 530, A115

Castro, N., Urbaneja, M. A., Herrero, A., et al. 2012, A\&A, 542, A79

Conti, P. S., \& Ebbets, D. 1977, ApJ, 213, 438

Crowther, P. A., Lennon, D. J., \& Walborn, N. R. 2006, A\&A, 446, 279

Davies, B., Kudritzki, R.-P., Plez, B., et al. 2013, ApJ, 767, 3

Ekström, S., Georgy, C., Eggenberger, P., et al. 2012, A\&A, 537, A146

Firnstein, M., \& Przybilla, N. 2012, A\&A, 543, A80

Girardi, L., Bressan, A., Bertelli, G., \& Chiosi, C. 2000, A\&AS, 141, 371

Heger, A., Langer, N., \& Woosley, S. E. 2000, ApJ, 528, 368

Hertzsprung, E. 1905, Z. wissenschaftliche Photographie, 3, 429

Humphreys, R. M., \& Davidson, K. 1979, ApJ, 232, 409

Hunter, I., Brott, I., Langer, N., et al. 2009, A\&A, 496, 841

Iben, Jr., I. 1967, ARA\&A, 5, 571

Kippenhahn, R., \& Weigert, A. 1990, Stellar Structure and Evolution

Köhler, K., Langer, N., de Koter, A., et al. 2014, A\&A, submitted

Kudritzki, R. P., Bresolin, F., \& Przybilla, N. 2003, ApJ, 582, L83

Langer, N. 1991, A\&A, 252, 669

Langer, N. 2012, ARA\&A, 50, 107

Langer, N., \& Kudritzki, R. P. 2014, A\&A, 564, A52

Langer, N., \& Maeder, A. 1995, A\&A, 295, 685

Lefever, K., Puls, J., \& Aerts, C. 2007, A\&A, 463, 1093

Lefever, K., Puls, J., Morel, T., et al. 2010, A\&A, 515, A74

Levesque, E. M., Massey, P., Olsen, K. A. G., et al. 2005, ApJ, 628, 973

Markova, N., Puls, J., Repolust, T., \& Markov, H. 2004, A\&A, 413, 693

Markova, N., Puls, J., Simón-Díaz, S., et al. 2014, A\&A, 562, A37

Martins, F., Schaerer, D., Hillier, D. J., et al. 2005, A\&A, 441, 735

Martins, F., Mahy, L., Hillier, D. J., \& Rauw, G. 2012, A\&A, 538, A39

Massey, P. 2003, ARA\&A, 41, 15

McErlean, N. D., Lennon, D. J., \& Dufton, P. L. 1999, A\&A, 349, 553

Meynet, G., \& Maeder, A. 2000, A\&A, 361, 101

Mokiem, M. R., de Koter, A., Puls, J., et al. 2005, A\&A, 441, 711

Nielsen, A. V. 1964, Centaurus, 9, 219

Nieva, M.-F. 2013, A\&A, 550, A26

Przybilla, N., Butler, K., \& Kudritzki, R. P. 2001, A\&A, 379, 936

Repolust, T., Puls, J., \& Herrero, A. 2004, A\&A, 415, 349

Russell, H. N. 1919, Proc. National Academy of Science, 5, 391

Sana, H., de Mink, S. E., de Koter, A., et al. 2012, Science, 337, 444

Schaller, G., Schaerer, D., Meynet, G., \& Maeder, A. 1992, A\&AS, 96, 269

Searle, S. C., Prinja, R. K., Massa, D., \& Ryans, R. 2008, A\&A, 481, 777

Simón-Díaz, S. 2010, A\&A, 510, A22

Simón-Díaz, S. 2014, in Proc. IAUS, 307 [arXiv : 1409. 2416]

Simón-Díaz, S., \& Herrero, A. 2014, A\&A, 562, A135

Simón-Díaz, S., Castro, N., Garcia, M., Herrero, A., \& Markova, N. 2011a, Bulletin de la Société Royale des Sciences de Liège, 80, 514

Simón-Díaz, S., Castro, N., Herrero, A., et al. 2011b, J. Phys. Conf. Ser., 328, 2021

Smith, N. 2014, ARA\&A, 52, 487

Soderblom, D. R. 2010, ARA\&A, 48, 581

Soubiran, C., Le Campion, J.-F., Cayrel de Strobel, G., \& Caillo, A. 2010, A\&A, 515, A111

Vandenberg, D. A. 1985, ApJS, 58, 711

Yoon, S.-C., Langer, N., \& Norman, C. 2006, A\&A, 460, 199

Yorke, H. W. 1986, ARA\&A, 24, 49 


\section{Appendix A: sHRD with rotating models}

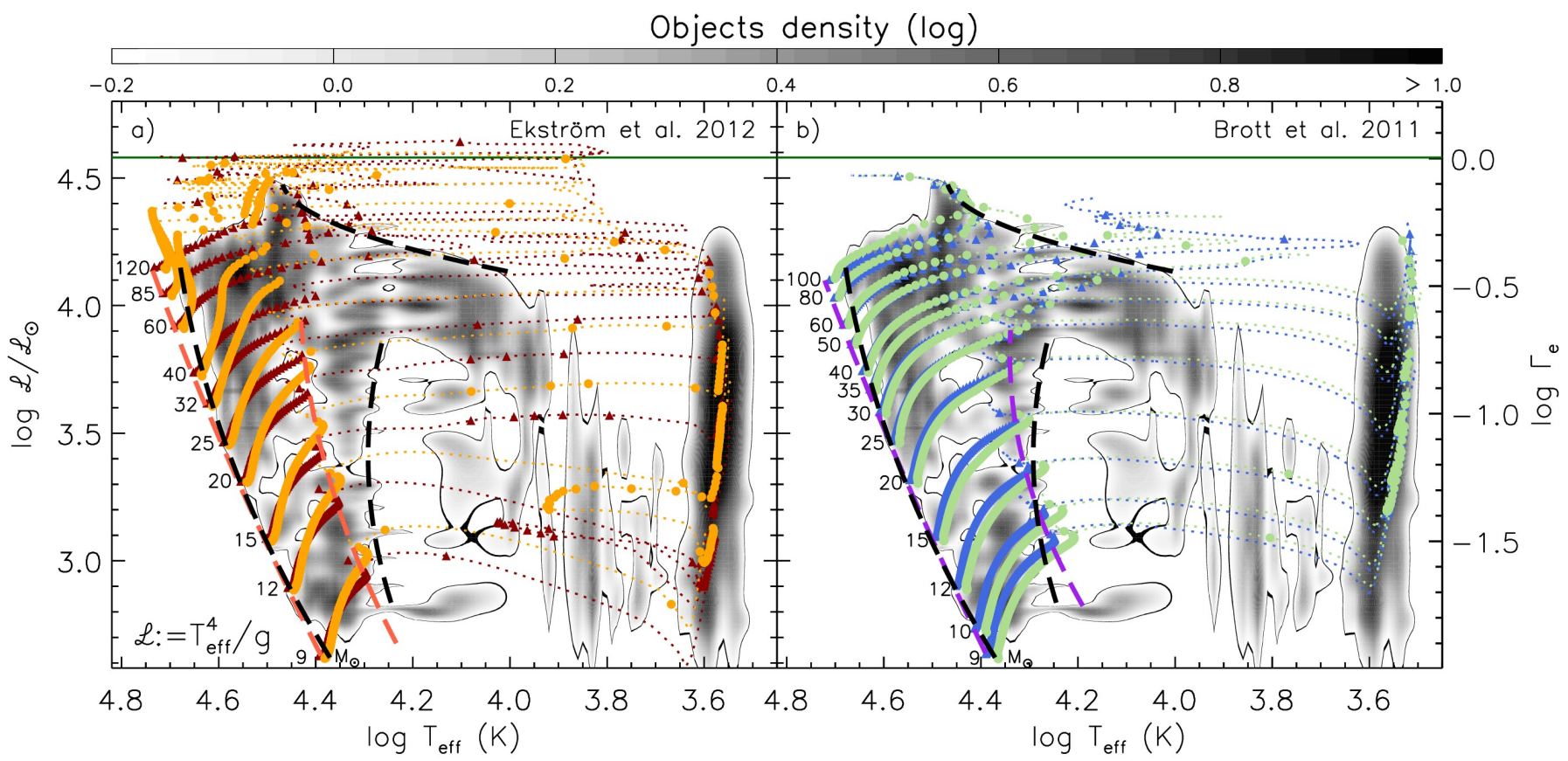

Fig. A.1. As in Fig. 1, but including rotating stellar evolution tracks: a) models of Ekström et al. (2012) with an initial rotational velocity of 40\% of critical rotation (orange dotted lines and dots) and; b) models of Brott et al. (2011) with an initial rotational velocity of $300 \mathrm{~km} \mathrm{~s}^{-1}$ (green dotted lines and dots). 